\title{
GaN LAYERS GROWN BY REACTIVE ION PLATING*
}

\author{
A. Żubka, R. DWiliński, B. Suchanek, W. Janik, A. WYSmolek,
} S. KWIaTKowski, M. KamińsKa

Institute of Experimental Physics, Warsaw University

Hoża 69, 00-681 Warszawa, Poland

and L. Shaginyan

Institute of Material Problems, Krzyżanowskowo 3, 252142 Kiev, Ukraine

GaN layers grown on ceramics, sapphire or $\mathrm{SiC}$ substrates using reactive ion plating method are presented. In reactive ion plating method gallium from a hot source reacts on a heated substrate with nitrogen partially ionized. Rutherford backscattering technique was applied to check the composition of the samples and gallium to nitrogen ratio was found to be close to one. However, Rutherford backscattering studies showed also a remarkable amount of unintentional impurities present in the layers. The structure of $\mathrm{GaN}$ was determined using reflection high-energy electron diffraction. It appeared that polycrystal and monocrystal can be grown, depending on growth conditions. Absorption spectra taken on the layers grown on sapphire showed a tail of band to band absorption starting at about $370 \mathrm{~nm}$. Carrier concentration was of the order of $10^{19}-10^{20} \mathrm{~cm}^{-3}$ at room temperature and did not change much with temperature decrease. No luminescence from the layers was detected, most probably due to high concentration of impurities.

PACS numbers: $68.55 . \mathrm{Gi}, 81.15 . \mathrm{Jj}$

\section{Introduction}

In the last few years one can observe an increasing interest in wide-gap semiconductors such as diamond, silicon carbide and nitrides. Gallium nitride, one of these compounds, has many features that make it promising material for future electronic and optoelectronic applications. The most important features are: direct band gap of $3.4 \mathrm{eV}$, complete solubility in aluminum nitride and indium nitride and stability at high temperatures - up to $450^{\circ} \mathrm{C}$. GaN-based optoelectronic devices are expected to be much more efficient than those made of $\mathrm{SiC}$ or diamond, since

*This work is supported by the State Committee for Scientific Research (Republic of Poland) grant No. 3 P407 07106. 
the last two semiconductors have indirect energy gap. Moreover, it is possible to both increase and decrease $\mathrm{GaN}$ band gap by dissolving certain amounts of aluminum- or indium nitride. GaN is already used to produce a commercial blue LED and it is expected that in close future nitrides will dominate short wavelength optoelectronic device market and will be used in blue and ultraviolet detectors, LEDs and laser diodes.

Typical ways to obtain GaN films are metal-organic chemical vapor deposition (MOCVD) and plasma-assisted molecular beam epitaxy (MBE) techniques $[1,2]$. Reactive ion plating (RIP) method although much older than the two others mentioned above is not as popular. In RIP method gallium from a hot source reacts with partially ionized nitrogen on a heated substrate. This relatively simple technique has some advantages. First of all, the costs of equipment and processes are low - one does not need ultrahigh vacuum like in MBE or expensive chemicals as in MOCVD. Secondly, the growth rate is much higher than in MBE. The third advantage is connected with the main problem of GaN film technology at the moment which is a need of good substrate. Therefore two types are used: silicon carbide or sapphire. Sapphire has the lattice mismatch of about $14 \%$ and $\mathrm{SiC}-$ much less of about $3.4 \%$. Very important advantage of RIP is the possibility of growth of GaN layers on SiC. All MOCVD films are grown on sapphire, because on the surface of $\mathrm{SiC}$ in the presence of nitrogen $\mathrm{Si}_{3} \mathrm{~N}_{4}$ compound is produced. At present homoepitaxy is practically not possible since GaN crystals are extremely hard to grow and their maximum size does not exceed a few millimeters.

\section{GaN deposition}

The growth of GaN layers was performed at Crystal Growth Laboratory, Warsaw University. The scheme of the applied growth system is presented in Fig. 1. Deposition took place in a vacuum chamber which was pumped down to $3 \times 10^{-5}$ torr and then filled with $\mathrm{N}_{2}$ gas up to $5 \times 10^{-3}$ torr. GaN was deposited on different types of substrates. Typically each process was performed with three types: $\mathrm{SiC}, \mathrm{Al}_{2} \mathrm{O}_{3}$ and ceramics (the dimensions were about $5 \times 5 \mathrm{~mm}$ ). The substrates were etched in boiling HF for 20 minutes and then rinsed in deionized water. Then they were mounted on a holder, which was heated by a halogen lamp to temperatures not higher than $800^{\circ} \mathrm{C}$. The temperature of the substrate was critical for the process, as the reaction between gallium and nitrogen took place only on the surface [3]. Metallic gallium was placed in a BN crucible of $10 \mathrm{~mm}$ diameter. The crucible was heated up to $1500^{\circ} \mathrm{C}$. This lead to gallium transport towards the substrate in atmosphere of ionized nitrogen.

The crucial thing in the applied growth method was to get nitrogen gas ionized. Only ionized nitrogen could react with gallium. Electromagnetic field of 13.56 $\mathrm{MHz}$ frequency (radio frequency - RF) induced in aluminum coil $(\approx 6 \mathrm{~cm}$ diameter) connected to a RF generator was used for this purpose. The coil was mounted in such a way that gallium coming from BN crucible went through it. Stabilization of proper nitrogen plasma pressure was the main problem in the setup. The pressure of nitrogen inside the chamber had to be above $2 \times 10^{-3}$ torr, otherwise no plasma occurs. On the other hand, it had to be kept as low as 


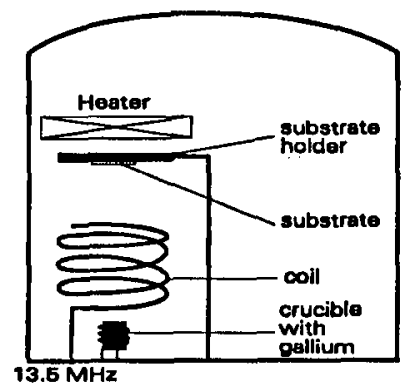

Fig. 1. Scheme of the deposition system.

possible to minimize contamination. Therefore, very precise control of $\mathrm{N}_{2}$ pressure was required. In the setup used the control of nitrogen plasma pressure was not good enough to obtain layers of reproducible properties. The other problem was that ionized nitrogen reacted not only with gallium but with everything inside the chamber. Therefore at present we are redesigning the chamber with better pressure control system and screens to reduce contamination caused by these reactions.

\section{Layer properties}

In RIP method the following parameters were important for the properties of the grown GaN layers: nitrogen pressure, substrate and gallium source temperatures, voltage and distance between gallium source and substrate, power of RF generator and time of deposition. Depending on the conditions we were able to grow thin or thick layers, monocrystals, polycrystals or amorphous GaN.
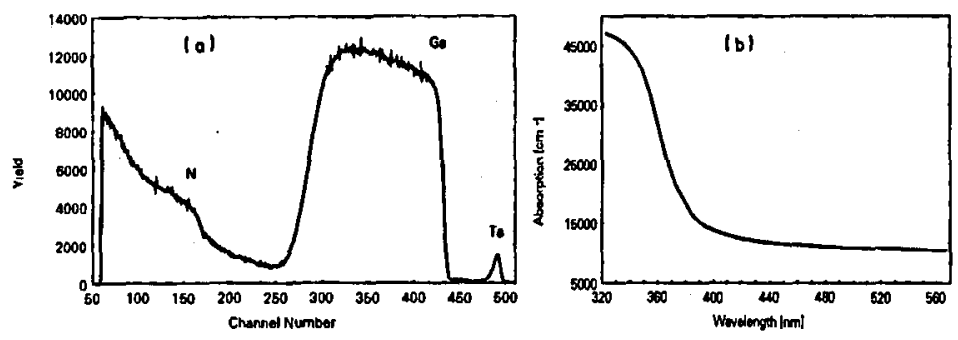

Fig. 2. (a) RBS spectrum of $\mathrm{GaN} / \mathrm{SiC}$ layer. Ta contamination visible. Ga/N ratio 0.99. (b) Absorption spectrum of GaN/sapphire layer.

Rutherford backscattering (RBS) was applied to check the composition of the layers. By this method the exact sample thickness could also be measured. For the grown layers gallium to nitrogen ratio close to one was obtained (Fig. 2a). However, RBS indicated also presence of high concentration of unintentional impurities, like Mn or Ta.

Further evidence that GaN film was grown was coming from the optical absorption experiments. In Fig. 2b a typical absorption spectrum of GaN grown on 

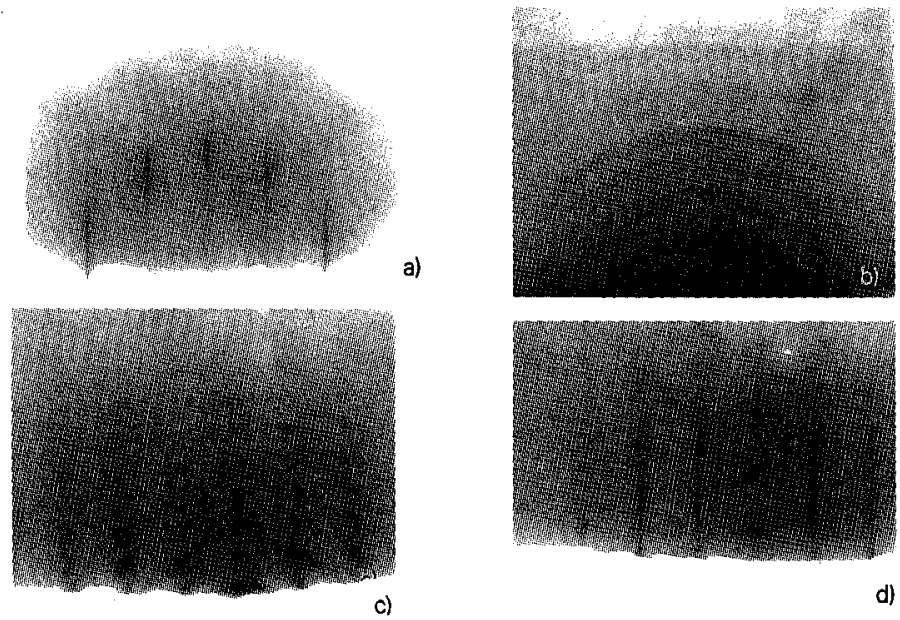

d)

Fig. 3. RHEED patterns of: (a) SiC substrate; (b) GaN polycrystal layer; (c) GaN polycrystal with texture; (d) $\mathrm{GaN}$ monocrystal.

sapphire is shown. The measurements were done using two-beam spectrophotometer CARY 5. In comparison with GaN absorption spectra published by others [4] the absorption edge is not as sharp but its energy position is very similar.

Reflection high-energy electron diffraction (RIIEED) was used to study structural properties of the layers. In Fig. 3 different pictures of RHEED patterns coming from: $\mathrm{SiC}$ monocrystals (substrate), $\mathrm{GaN} / \mathrm{SiC}$ polycrystal, $\mathrm{GaN} / \mathrm{SiC}$ polycrystal with texture and $\mathrm{GaN} / \mathrm{SiC}$ monocrystal are presented. As seen from Fig. 3 the wurtzite-type structure of GaN gave different image than the surface of $6 \mathrm{H}-\mathrm{SiC}$. Typical images of wurtzite structure were observed both from mono- and polycrystals. It was found that monocrystal layers could be obtained in substrate temperature range of $620^{\circ} \mathrm{C}$ to $730^{\circ} \mathrm{C}$ and for $\mathrm{N}_{2}$ pressure in the range of $1 \times 10^{-3}$ torr to $8 \times 10^{-3}$ torr.

The Hall effect was measured on the grown GaN films with four indium contacts. Very low mobility (of the order of $2 \mathrm{~cm}^{2} / \mathrm{Vs}$ ) and high carrier concentration $\left(10^{19}-10^{20} \mathrm{~cm}^{-3}\right)$ were found. These values were similar to some reported in literature [5], although layers with higher mobilities could be also obtained by MBE or MOCVD methods.

No photoluminescence from the layers was detected.

\section{Résumé}

In this paper we showed that RIP can be used to obtain GaN films of different structure: from amorphous to monocrystals. The properties of these are not satisfying yet for optoelectronic application since no photoluminescence was observed, most probably because of high concentration of unintentional impurities. The improvement of layer properties can be expected when our new deposition setup, which will give higher purity of the layers, better control of process parameters and good stability of growth conditions will be used. 


\section{Acknowledgments}

Herewith we would like to thank Dr. Kazimierz Regiński for a lot of help with RHEED measurements.

\section{References}

[1] S. Nakamura, Jpn. J. Appl. Phys. 30, 1620 (1991).

[2] M.J. Paisley, Z. Sitar, J.B. Posthill, R.F. Davis, J. Vac. Sci. Technol. A 7, 701 (1989).

[3] A.Yu. Dyban, I.A. Kossko, A.N. Pilyankevich, L.R. Shaginyan, Surface. Physics, chemistry, mechanics, Soviet Academy of Science, Vol. 2, 1990, p. 67.

[4] B.-C. Chung, M. Gershenzon, J. Appl. Phys. 72, 651 (1992).

[5] M. Ilegems, R. Dingle, J. Appl. Phys. 44, 4234 (1973). 